Portland State University

PDXScholar

Electrical and Computer Engineering Faculty

Publications and Presentations

$1-1-2003$

\title{
Source motion mitigation for adaptive matched field processing
}

Lisa M. Zurk

Portland State University

Nigel Lee

James Ward

Follow this and additional works at: https://pdxscholar.library.pdx.edu/ece_fac

Part of the Electrical and Computer Engineering Commons

Let us know how access to this document benefits you.

Citation Details

Zurk, L. M., Lee, N., \& Ward, J. (2003). Source motion mitigation for adaptive matched field processing. The Journal of the Acoustical Society of America,113, 2719.

This Article is brought to you for free and open access. It has been accepted for inclusion in Electrical and Computer Engineering Faculty Publications and Presentations by an authorized administrator of PDXScholar. Please contact us if we can make this document more accessible: pdxscholar@pdx.edu. 


\title{
Source motion mitigation for adaptive matched field processing $^{\text {a) }}$
}

\author{
Lisa M. Zurk, ${ }^{\text {b) }}$ Nigel Lee, and James Ward \\ MIT Lincoln Laboratory, 244 Wood Street, Lexington, Massachusetts 02420
}

(Received 30 November 2001; revised 23 December 2002; accepted 23 December 2002)

\begin{abstract}
Application of adaptive matched field processing to the problem of detecting quiet targets in shallow water is complicated by source motion, both the motion of the target and the motion of discrete interferers. Target motion causes spreading of the target peak, thereby reducing output signal power. Interferer motion increases the dimensionality of the interference subspace, reducing adaptive interference suppression. This paper presents three techniques that mitigate source motion problems in adaptive matched field processing. The first involves rank reduction, which enables adaptive weight computation over short observation intervals where motion effects are less pronounced. The other two techniques specifically compensate for source motion. Explicit target motion compensation reduces target motion mismatch by focusing snapshots according to a target velocity hypothesis. And time-varying interference filtering places time-varying nulls on moving interferers not otherwise suppressed by adaptive weights. The three techniques are applied to volumetric array data from the Santa Barbara Channel Experiment and are shown to improve output signal-to-background-plus-noise ratio by more than $3 \mathrm{~dB}$ over the standard minimum-variance, distortionless response adaptive beam-former. Application of the techniques in some cases proves to be the difference between detecting and not detecting the target. (C) 2003 Acoustical Society of America. [DOI: 10.1121/1.1561817]
\end{abstract}

PACS numbers: 43.60.Gk, 43.30.Wi [JCB]

\section{INTRODUCTION}

This paper presents and applies three "motion mitigation" techniques for improving adaptive matched field processing detection of quiet, moving targets in shallow-water environments.

Detection and localization of targets in shallow-water environments is a challenging problem for which it is wellknown that plane-wave beamforming is inadequate because channel-specific acoustic multipath is not accounted for. ${ }^{1} \mathrm{By}$ contrast, matched field processing (MFP) accounts for coherent acoustic multipath in shallow water by employing a propagation model to construct appropriate steering (or "replica") vectors. Conventional (nonadaptive) matched field processing tends to suffer from beampatterns with high sidelobes, which can obscure quiet target detection in the presence of strong interferers. Adaptive matched field processing (AMFP) reduces interferer sidelobes by computing datadependent weight vectors based on sample covariance matrix (SCM) inversion. ${ }^{2-4}$

It is well known, however, that AMFP performance de-

\footnotetext{
a) Portions of this work were presented in "3D adaptive matched field processing for a moving source in a shallow water channel," Proceedings of the IEEE Oceans '99 Conference, Seattle, WA, September 1999; in "Evaluation of reduced-rank adaptive matched field processing algorithms for passive sonar detection in a shallow-water environment," Proceedings of the 33rd Asilomar Conference, Pacific Grove, CA, November 1999; in "Adaptive matched field processing for a moving target in a noisy shallow water channel," Proceedings of the Adaptive Sensor Array Processing (ASAP) Workshop, Lexington, MA, March 2000; and in "Interference rejection for passive sonar using prior information with adaptive matched field processing," Fifth European Conference on Underwater Acoustics, Lyon, France, July 2000.

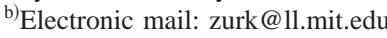

grades quickly in less-than-ideal conditions. ${ }^{1,3}$ In particular, moving sources (both targets and interferers) can degrade AMFP performance severely, especially for large arrays with small beamwidths. ${ }^{5}$ Target motion spreads target energy across several beams, reducing output signal power and resulting in poorer target detection and localization. Interferer motion increases the dimensionality of the interference subspace, reducing adaptive interference suppression and again resulting in poorer weak target detection.

One way to mitigate source motion is to apply rank reduction, which allows adaptive weight computation over shorter observation intervals where sources move less and are quasi-stationary. In much of the literature, rank reduction for AMFP is performed in eigenvector space. ${ }^{6-8}$ The drawback to this is that eigenvectors have no inherent physical basis, so eigenvector-based rank reduction is usually achieved with no regard for information provided by the propagation physics. By contrast, several authors ${ }^{9-11}$ have shown the utility of transforming the data into acoustic mode space, with a physically based modal basis; mode-space rank reduction to date, however, still employs eigenvectors. ${ }^{11}$ The first motion mitigation technique presented here performs mode-based rank reduction (MBRR) by selecting the reduced-rank mode space according to physical considerations. In addition to the general benefits of rank reduction, this technique provides both filtering of surface interferers and broadening of beamwidths in the output beamformer, both of which further mitigate motion effects. MBRR does require accurate computation of the acoustic modes, so it is somewhat sensitive to environmental mismatch and it requires a capable array; this is discussed further in Sec. IV.

For processing over long observation intervals (in order 
to minimize adaptive bias $\operatorname{loss}^{2}$ ), various techniques seek to address either target motion (by compensation) or interferer motion (by filtering). In the former category, the concept of matched-field tracking ${ }^{12,13}$ has been proposed to mitigate target motion effects by adjusting the matched field replica vectors according to a target velocity hypothesis; however, matched field tracking can only produce nonadaptive output. By contrast, the second motion mitigation technique presented here performs explicit target motion compensation (ETMC) by focusing the data (snapshots), not the replicas, according to the target velocity hypothesis. This allows computation of target-motion-compensated adaptive output. The concept of focusing snapshots is found in broadband source localization problems, ${ }^{14,15}$ but there the focusing is done in frequency (for wideband but stationary sources), not in space (as done in MFT and ETMC for narrowband but moving sources).

To address the interference motion problem over long observation times, data-based, time-varying interference filtering has been proposed previously in other contexts ${ }^{16,17}$ and has recently been proposed for matched field processing. ${ }^{18-21}$ The idea is to filter moving interferers, which are not effectively nulled by normal adaptive processing, on a snapshot-by-snapshot basis, under the assumption that the principal eigenvectors for each snapshot represent the interferers to be filtered. This technique fails, however, whenever the target itself is loud relative to the interferers. The third motion mitigation technique presented here performs model-based, time-varying interference filtering (MTIF) that makes use of external track information to place location-based nulls on interferers. Note that MTIF does not require association of the interferer with eigenvectors of the data; however, it does require accurate modeling of the interference space and is thus sensitive to (environment and track) mismatch. A hybrid algorithm is also presented that combines information from both the model-based and databased techniques.

All three of the proposed techniques are demonstrated on vertical line array (VLA) data obtained from the Santa Barbara Channel Experiment (SBCX). ${ }^{22}$ Section II presents the basic AMFP framework used in this work. Section III describes in detail the three techniques of improving AMFP performance on moving sources (reduced-rank mode space processing, explicit target motion compensation, and timevarying interference filtering). Section IV shows the results of applying the techniques to SBCX data and the improvement they provide over standard AMFP output and over existing motion mitigation techniques. Finally, Sec. V summarizes the conclusions of this work and suggests some areas for further work.

\section{ADAPTIVE MATCHED FIELD PROCESSING FRAMEWORK}

This section presents the basic AMFP framework used throughout the paper.

Defining $\Theta=(r, \phi, z)$ as the three-dimensional spatial position in range, azimuth, and depth, the MFP output for an $N$-element array at frequency $f_{0}$, time $t_{0}$, and direction $\Theta$ can be written as

$$
P\left(f_{0}, t_{0}, \Theta\right)=\vec{w}^{H}\left(f_{0}, t_{0}, \Theta\right) \hat{\mathbf{K}}\left(f_{0}, t_{0}\right) \vec{w}\left(f_{0}, t_{0}, \Theta\right),
$$

where $\vec{w}\left(f_{0}, t_{0}, \Theta\right)$ is the $N \times 1$ weight vector based on the corresponding replica vector $\vec{v}\left(f_{0}, t_{0}, \Theta\right)$, and where $\hat{\mathbf{K}}\left(f_{0}, t_{0}\right)$ is the $N \times N$ sample covariance matrix discussed in the following. For conventional matched field processing $(\mathrm{CMFP}),{ }^{23}$ the weights in Eq. (1) are normalized replica vectors:

$$
\vec{w}_{c}\left(f_{0}, t_{0}, \Theta\right)=\vec{v}\left(f_{0}, t_{0}, \Theta\right) / N,
$$

where the replica vector is normalized such that $\left|\vec{v}\left(f_{0}, t_{0}, \Theta\right)\right|^{2}=N$, so that the CMFP weight vector $\vec{w}_{c}(\cdot)$ in Eq. (2) achieves unity gain on target.

Adaptive matched field processing computes a weight vector that is dependent on the sample covariance matrix as well as the replica vector. The AMFP results in this paper are based on the high-resolution minimum-variance, distortionless-response (MVDR) filter. ${ }^{2,3}$ The diagonally loaded MVDR (or MVDR-DL) weight vector is given by

$$
\vec{w}_{m}(\cdot)=\frac{\left(\hat{\mathbf{K}}\left(f_{0}, t_{0}\right)+\sigma_{d}^{2}(\cdot) \mathbf{I}\right)^{-1} \vec{v}(\cdot)}{\vec{v}(\cdot)^{H}\left(\hat{\mathbf{K}}\left(f_{0}, t_{0}\right)+\sigma_{d}^{2}(\cdot) \mathbf{I}\right)^{-1} \vec{v}(\cdot)},
$$

where the MVDR weight vector $\vec{w}_{m}(\cdot)=\vec{w}_{m}\left(f_{0}, t_{0}, \Theta\right)$ is based on the replica vector $\vec{v}(\cdot)=\vec{v}\left(f_{0}, t_{0}, \Theta\right)$ and a position-dependent diagonal load level $\sigma_{d}^{2}(\cdot)$ $=\sigma_{d}^{2}\left(f_{0}, t_{0}, \Theta\right)$. The load level is chosen to be large enough to satisfy a white noise gain constraint ${ }^{4}$ (WNGC)

$$
\vec{w}_{m}^{H}(\cdot) \vec{w}_{m}(\cdot) \leqslant \frac{\beta}{N},
$$

where the constant $\beta$ is a "relaxation" parameter, defined as the factor by which the weight norm in Eq. (4) is allowed to exceed the "white noise gain" of $1 / N$ [which is the value of $\vec{w}_{m}^{H}(\cdot) \vec{w}_{m}(\cdot)$ with no loading when $\left.\hat{\mathbf{K}}\left(f_{0}, t_{0}\right)=\mathbf{I}\right]$. The WNGC load level that just satisfies Eq. (4) can be efficiently found through iterative searching, ${ }^{8}$ which is the method employed here. Diagonally loading the MVDR weight vector minimizes the effects of poorly estimated components of the $\operatorname{SCM~} \hat{\mathbf{K}}\left(f_{0}, t_{0}\right)$ (due to insufficient snapshots, for example) as well as the effects of target self-nulling due to mismatch.

MFP weights are computed over a finite set of spatial coordinates $\Theta$, and MFP results are typically displayed via a spatial ambiguity surface measuring MFP output power [from Eq. (1) above] versus spatial variables (range, depth, and/or bearing).

In this paper, the $N \times N$ sample covariance matrix $\hat{\mathbf{K}}\left(f_{0}, t_{0}\right)$ in Eq. (3) is computed using a time average of snapshots taken from FFT data at the frequency of interest:

$$
\hat{\mathbf{K}}\left(f_{0}, t_{0}\right)=\frac{1}{L} \sum_{l=-L / 2}^{L / 2-1} \vec{x}\left(f_{0}, t_{0}+l \Delta t\right) \vec{x}\left(f_{0}, t_{0}+l \Delta t\right)^{H},
$$

where $\vec{x}(f, t)$ denotes the $N \times 1$ snapshot computed at frequency $f$ and time $t, f_{0}$ is the center frequency, $t_{0}$ is the "center time," and $L$ is the number of snapshots. With nonoverlapping FFT windows, the estimate in Eq. (5) requires an observation period of $T=L \Delta t$ for each covariance computation, where $\Delta t$ is the FFT window length. 
The baseline AMFP output for this paper is the power output in Eq. (1) computed using the MVDR-DL weight vector in Eq. (3) and the sample covariance matrix in Eq. (5). In all subsequent sections of this paper, the dependence of $\vec{v}$, $\vec{w}, \hat{\mathbf{K}}$, and $\mathbf{P}$ on the center frequency $f_{0}$ and center time $t_{0}$ is suppressed and assumed implicitly, except where needed for clarity.

\section{AMFP MOTION MITIGATION}

\section{A. Quantifying motion effects for MFP}

The effect of source motion on MFP detection is best understood by considering the intrinsic cell size of a matched field processor (equivalent to the beamwidth of a plane wave beamformer). The width of an MFP "cell" in a given spatial dimension (range, depth, or bearing) is defined as the distance between the half-power points of the CMFP beampattern in that dimension, where the gain of the beampatter is one half ( $3 \mathrm{~dB}$ below) its maximum value. MFP achieves relatively fine resolution due to the deterministic phasing of acoustic modes. Rough expressions of the range and depth cell sizes can be obtained by considering the span of horizontal and vertical wavenumbers of the propagating modes. Letting $k_{M}$ denote the horizontal wavenumber and $k_{z_{M}}$ the vertical wavenumber of the $M$ th mode (representing the highest propagating mode with significant energy), the following are approximate expressions for the MFP range and depth cell sizes $C_{R}$ and $C_{Z}$, respectively: ${ }^{9,24,25}$

$$
\begin{aligned}
& C_{R}=\frac{2 \pi}{k_{1}-k_{M}}, \\
& C_{Z}=\frac{\pi}{k_{z_{M}}},
\end{aligned}
$$

where $k_{1}$ is the wavenumber of the first mode (the mode that propagates nearly horizontally). The MFP bearing cell size is well-approximated by the corresponding expressions in plane wave beamforming:

$$
\begin{aligned}
& C_{\phi} \approx \lambda / L \quad \text { (near broadside) } \\
& C_{\phi} \propto \sqrt{\lambda / L} \quad \text { (near endfire), }
\end{aligned}
$$

where $L$ is the horizontal array extent and $\lambda$ is the acoustic wavelength. Note that a straight VLA has no horizontal extent, but a tilted VLA has a small horizontal extent.

The effect of target motion over a given observation time $T$ is to disperse the energy of the target in the MFP ambiguity surface across several MFP cells. One can define target "motion loss" as the loss in peak target power due to target motion, with the loss computed relative to the power of a stationary target. If the target is thought to be at position $\Theta$ but in reality transits through $\Theta$ over time, the motion loss (in $\mathrm{dB}$ ) as a function of $\Theta$ is given by

$$
M L(\Theta)=-10 \log _{10}\left[\frac{1}{L} \sum_{l=1}^{L} \cos ^{2}\left(\vec{x}_{t_{l}}, \vec{w}(\Theta)\right)\right],
$$

where

$$
\cos ^{2}\left(\vec{x}_{t_{l}}, \vec{w}(\Phi)\right) \triangleq \frac{\left|\vec{w}^{H}(\Theta) \vec{x}_{t_{l}}\right|^{2}}{|\vec{w}(\Theta)|^{2}\left|\vec{x}_{t_{l}}\right|^{2}}
$$

represents the beam offset between the target signature $\vec{x}_{t_{l}}$ at time $t_{l}$ and the MFP weight vector $\vec{w}^{H}(\Theta)$ for the assumed target position $\Theta$. For a stationary target, assuming no other sources of mismatch, $\cos ^{2}\left(\vec{x}_{t}, \vec{w}(\Theta)\right)=1$ and $M L(\Theta)=0 \mathrm{~dB}$. For CMFP, expression (11) reduces to $\cos ^{2}\left(\vec{x}_{t}, \vec{v}(\Theta)\right)$, because the weight vector $\vec{w}_{c}(\Theta)$ is a scalar multiple of the replica vector $\vec{v}(\Theta)$. $M L(\Theta)$ quantifies an upper bound to signalto-background-plus-noise-ratio (SBNR) loss due to target motion, specifically the "signal" portion of SBNR. [SBNR loss due to target motion may be less than $M L(\Theta)$ if, to begin with, the weight vector $\vec{w}(\Theta)$ is mismatched with the assumed (stationary) target at position $\Theta$.] This loss can be estimated by using Eqs. (6) and (7) to calculate the number of cells transited by the target during the observation time $T$, which may then be used to bound the allowable radial velocity. For example, in the SBCX environment, the $3 \mathrm{~dB}$ range cell size in Eq. (6) is approximately $10 \lambda$. If a source in this environment moves in range with a radial velocity greater than $10 \lambda / T$, its motion loss in Eq. (10) is greater than $3 \mathrm{~dB}$.

The effect of interferer motion over a given observation time $T$ is to spread interferer energy across the eigenvalue spectrum of the sample covariance matrix. The result of this is that moving interferers consume adaptive degrees of freedom (DOF) and limit the adaptive nulling capability of the AMFP weight vector (3). The number of adaptive DOF consumed by a moving interferer is roughly equivalent to the number of resolution cells [given again by Eqs. (6) and (7) above] that the interferer transits during the observation time.

\section{B. Motion mitigation by mode-based rank reduction (MBRR)}

One philosophy for mitigating source motion is to compute AMFP weights over shorter observation intervals where sources-both target and interferers-move less and are quasi-stationary, thus limiting motion loss. Shorter observation intervals, however, mean fewer data snapshots for SCM computation, and it is well known that MVDR power output is biased low if not enough snapshots are available.,26,27 Rank reduction is one method of decreasing the number of snapshots needed. (There is a limit, however, as to how short the observation time $T$ can be; this limit is determined by the number of snapshots needed to estimate all discrete sources in the data, including the target.)

Rank reduction for AMFP involves computing a reduced-rank approximation of the sample covariance matrix $\hat{\mathbf{K}}$ in Eq. (5). Most commonly, this is done using the eigenvector (EV) decomposition of $\hat{\mathbf{K}}$, given by

$$
\hat{\mathbf{K}}=\mathbf{U} \mathbf{\Sigma} \mathbf{U}^{H}=\sum_{i=1}^{N} \sigma_{i}^{2} \vec{u}_{i} \vec{u}_{i}^{H},
$$

where $\mathbf{U}=\left[\vec{u}_{1} \vec{u}_{2} \cdots \vec{u}_{N}\right]$ is an orthogonal matrix whose columns are comprised of the eigenvectors $\vec{u}_{i}$ of $\hat{\mathbf{K}}$ and $\boldsymbol{\Sigma}$ $=\operatorname{diag}\left(\sigma_{1}^{2}, \sigma_{2}^{2}, \ldots, \sigma_{N}^{2}\right)$ is a diagonal matrix whose diagonal elements are comprised of the eigenvalues $\sigma_{i}^{2}$ of $\hat{\mathbf{K}}$. Transformation from phone space (snapshots $\vec{x}$ ) to EV space is represented as $\vec{x}_{e}=\mathbf{T}_{e} \vec{x}$, where the $N \times N$ eigenvector transformation matrix is given by $\mathbf{T}_{e}=\mathbf{U}^{H}$. Eigenvectorspace rank reduction is then achieved by retaining $P$ out of $N$ 
elements of $\vec{x}_{e}$, which is equivalent to applying an eigenvector filter $\Phi_{e}: \vec{x}_{e_{R}}=\Phi_{e} \vec{x}_{e}=\Phi_{e} \mathbf{T}_{e} \vec{x}$, where $\Phi_{e}$ is a diagonal matrix with 1's (corresponding to retained eigenvectors) and 0 's (corresponding to filtered eigenvectors) as its diagonal entries.

The drawback to EV-based rank reduction is that eigenvectors have no inherent physical basis, so the rank reduction is usually achieved with no regard for information provided by the propagation physics. The most common example of eigenvector-based rank reduction is to retain the eigenvectors corresponding to the largest eigenvalues, which is essentially what is done in the well-known dominant mode rejection (DMR) algorithm. ${ }^{6}$ Other techniques exist for EV-based rank reduction in which the criterion for retaining eigenvectors is based at least in part on correlation with the replica vector look direction; examples of this include the signal coherence criterion $^{8}$ and the direct-form-cross-spectral metric, which was proposed and examined by the authors. ${ }^{28,29}$ However, the latter techniques require computation of a different reduced-rank EV subspace with each look direction and are thus computationally expensive.

AMFP rank reduction that is both physically based and computationally simple is made possible by transformation of both data and replicas into acoustic mode space. The acoustic mode functions are the mathematical descriptions of the physical phenomena assumed by normal mode propagation models, and the number of independent mode functions is limited to the number of propagating modes. Further, for a fully spanning, upright VLA, the propagating acoustic mode functions form an approximately orthonormal basis (note, however, that vertical aperture is essential to the formation of an orthonormal modal basis).

Modal decomposition makes use of the normal mode expansion of the acoustic pressure field $p(r, z)$ at receiver range $r$ and depth $z$ due to a source at depth $z_{s}$, which is approximated (in the far field) by

$$
p(r, z)=\frac{j}{\rho\left(z_{s}\right) \sqrt{8 \pi}} e^{-j \pi / 4} \sum_{i=1}^{M} \Psi_{i}\left(z_{s}\right) \Psi_{i}(z) \frac{e^{j k_{i} r}}{\sqrt{k_{i} r}},
$$

where $\Psi_{i}(\cdot)$ are the acoustic mode functions, $\rho\left(z_{s}\right)$ is the water density at the source depth, $k_{i}$ is the horizontal wavenumber associated with the $i$ th mode, and $M$ is the number of "propagating" modes for which $k_{i}$ does not have significant imaginary part. The expression in Eq. (13) may be rewritten as

$$
p(r, z)=\sum_{i=1}^{M} \alpha_{i}\left(z_{s}\right) f_{i}(r, z)
$$

where the constants $\alpha_{i}\left(z_{s}\right)$ may be thought of as coefficients in the modal expansion,

$$
\alpha_{i}\left(z_{s}\right)=\frac{j e^{-j \pi / 4}}{\rho\left(z_{s}\right) \sqrt{8 \pi}} \Psi_{i}\left(z_{s}\right)
$$

and where the functions $f_{i}(r, z)$ are the attenuated acoustic mode functions:

$$
f_{i}(r, z)=\Psi_{i}(z) \frac{e^{j k_{i} r}}{\sqrt{k_{i} r}} .
$$

To account for array tilt, the range $r$ in Eq. (16) may be replaced by an "adjusted" range $r+\Delta r$, with the elementdependent offset $\Delta r$ varying for each hydrophone (i.e., for each receiver depth $z$ ).

In vector notation, Eq. (14) may be rewritten as

$$
\vec{p}=\left(\mathbf{F}_{m} \odot \Gamma\right) \vec{a},
$$

where $\mathbf{F}_{m}$ is the $N \times M$ modal decomposition matrix whose $i$ th column is the attenuated mode function $f_{i}(r, z)$ in Eq. (16), sampled at the depths of the array hydrophones; where $\odot$ stands for element-by-element multiplication; where $\Gamma$ is a matrix of phase terms that takes into account the effects of array tilt; and where $\vec{a}$ is the $M \times 1$ vector of mode coefficients $\alpha_{i}$. Equivalently, the transformation of an $N \times 1$ "phone-space" data snapshot $\vec{x}$ to an $M \times 1$ mode-space snapshot $\vec{x}_{m}$ can be written as

$$
\vec{x}_{m}=\mathbf{T}_{m} \vec{x},
$$

where the $\mathbf{T}_{m}$ is the $M \times N$ mode transformation matrix computed as

$$
\mathbf{T}_{m}=\left\{\left(\mathbf{F}_{m} \odot \Gamma\right)^{H}\left(\mathbf{F}_{m} \odot \Gamma\right)\right\}^{-1}\left(\mathbf{F}_{m} \odot \Gamma\right)^{H} .
$$

The transformation matrix $\mathbf{T}_{m}$ in Eq. (19) is essentially that proposed in previous papers on mode-space MFP. ${ }^{9,11}$ The inverse in Eq. (19) can be unstable, especially if $M>N$ and $\mathbf{F}_{m}^{H} \mathbf{F}_{m}$ is less than full rank; in this case, small or zero eigenvalues of $\mathbf{F}_{m}^{H} \mathbf{F}_{m}$ may be dropped in order to compute the (pseudo)inverse. ${ }^{11}$

The first of three motion mitigation techniques proposed here is mode-based rank reduction (MBRR). This is achieved by retaining $P$ out of $M$ elements of $\vec{x}_{m}$ in Eq. (18), which is equivalent to applying a mode filter $\Phi_{m}$ :

$$
\vec{x}_{m_{R}}=\Phi_{m} \vec{x}_{m}=\Phi_{m} \mathbf{T}_{m} \vec{x},
$$

where $\Phi_{m}$ is a diagonal matrix with 1's (corresponding to retained modes) and 0's (corresponding to filtered modes) as its diagonal entries. For example, $\Phi_{m}=\operatorname{diag}(1, \ldots, 1,0, \ldots, 0)$ retains the first few (lower-order) modes and filters the rest.

MBRR can succeed where EV-based techniques do not because the mode functions have physical structure that can be exploited to separate submerged targets and surface interferers. (Successful eigenvector separation of nonorthogonal sources requires different source powers, which will not always be the case. Equal-power nonorthogonal sources will not be separated well using eigenvectors.) For example, because only higher-order modes are strongly excited at the water surface and because lower-order modes often contain a significant portion of the energy for sources at depth, one potentially effective MBRR technique is to retain only the lower-order modes (and to filter the higher-order modes). Of course, the success of mode-based surface-submerged source separation may vary with environment.

In addition to allowing adaptive processing with fewer snapshots, MBRR thus has the added benefit of filtering surface energy while not suffering significant signal gain degradation for targets at depth. Further, filtering of the higher- 
order modes has the effect of increasing the intrinsic MFP range cell size by decreasing the denominator of Eq. (6). Thus, AMFP motion effects are further mitigated, as both target and interferers transit fewer mode-space MFP cells than phone-space MFP cells, resulting in less target motion loss and fewer interferer DOF. It is also true, however, that the increased cell size results in decreased source localization accuracy.

\section{Motion mitigation by explicit target motion compensation (ETMC)}

A second philosophy for mitigating source motion is to compute AMFP weights over long observation intervals $T$ but to compensate explicity for source motion. Processing over long observation intervals, if done properly, increases the accuracy of the adaptive weight computation and increases incoherent integration gain. Over long observation intervals, however, both target and interferer motion have to be accounted for.

The second of three motion mitigation techniques proposed here is explicit target motion compensation (ETMC), which combats target motion loss over long observation intervals. This is accomplished by adjusting the amplitude and phase across each data snapshot $\vec{x}\left(f_{0}, t_{l}\right)$ in Eq. (5) so that the target appears stationary. It is important to note that ETMC (as well as other target motion compensation techniques) compensates for the motion of a single source; it cannot effectively compensate for different motions of multiple sources. The amplitude/phase adjustment is determined by applying a velocity hypothesis to the target to predict the target position at each time $t_{l}$, and then by comparing the target replica vector at each position (computed via a propagation model) with the replica vector at a chosen, "focus" position. Assuming uncorrelated sensor data, the adjustment for the $k$ th sensor at time $t_{l}$ is given by

$$
\Psi_{l}(k)=e^{-j \Delta \phi_{l}(k)} \Delta \alpha_{l}(k),
$$

where $\Delta \phi_{l}(k)$ is the phase difference between the response of the $k$ th hydrophone for the predicted target position at time $t_{l}$ and the response of the $k$ th hydrophone for the target focus position; and where $\Delta \alpha_{l}(k)$ is the corresponding target amplitude ratio. Computation of the correct compensations $\Delta \phi_{l}$ and $\Delta \alpha_{l}$ thus requires both an accurate target velocity hypothesis and accurate propagation modeling.

Once each snapshot has been "compensated," the resulting covariance matrix contains the signature of a target that has been "focused" to the focus point. The focus point is any position along the target track (during the observation interval) to which motion will be compensated; a single focus point is chosen for a given observation interval $T$. Perfect compensation produces output equivalent to that for a stationary target at the focus point; this theoretically recovers whatever target motion loss has occurred during the observation interval. In this paper, the predicted position of the target in the middle of the observation period is chosen as the focus point.

It is important to distinguish ETMC with matched field tracking (MFT) algorithms, ${ }^{12,13}$ which adjust the replica vectors $\vec{v}$ according to the target velocity hypothesis, while keeping the data (snapshots and SCM) fixed. Because the adaptive weight computation in Eq. (3) requires multiple snapshots for a given replica vector, target motion compensation for adaptive MFP requires adjustment of the data while keeping the replica vectors fixed. The latter is what is done in ETMC. Another significant difference is computational: MFT requires compensation of every replica vector with each snapshot in time, whereas ETMC requires only the adjustment of the snapshot itself. Again, it is important to note that both techniques can only compensate for the motion of a single source.

\section{Motion mitigation by model-based, time-varying interference filtering (MTIF)}

The last of three motion mitigation techniques proposed here is model-based, time-varying interference filtering (MTIF), which combats interference motion loss over long observation intervals. Interference filtering involves spatial filtering of an interferer, in which the data is projected onto a subspace that is orthogonal to the (estimated) interference subspace. Because interferers move, the rank of the interference subspace estimate over the entire observation interval $T$ can be quite large, and filtering of the data by this "longtime" interference subspace estimate may result in undesirable reduction of the target peak. The solution to this problem is to apply a time-varying spatial filter to the data that removes an instantaneous estimate of the interference subspace at each snapshot; the instantaneous subspace is likely low-rank because it is estimated over a very short observation time.

The spatial interference filter is the orthogonal projection complement $\mathbf{I}-\Phi\left(\Phi^{H} \Phi\right)^{-1} \Phi^{H}$ of the interference subspace estimate $\Phi$. This filter is applied to each snapshot, and the filtered snapshot is then target-motion-compensated as detailed earlier. The resulting filtered and compensated snapshot is then used as in Eq. (5) to compute the sample covariance matrix $\hat{\mathbf{K}}$.

Clearly, the most important aspect of the interference filtering technique is estimating the interference subspace. Data-based estimation assumes that the interferer is the strongest source in the data and is captured by the principal eigenvector(s) of the "instantaneous covariance matrix" computed for each snapshot. By contrast, MTIF makes use of external track information for the interferer to build a location-based interference subspace estimate. Hybrid estimation combines information from both. The three methods are detailed in the following.

\section{Data-based interference filtering}

The data-based method of interference filtering (similar to what Cox terms "multi-rate adaptation" ${ }^{18}$ ) assumes that the interference is strong and can be estimated with a small number of snapshots $J$, fewer than the total number of snapshots $L$. At each time $t_{l}$, an instantaneous covariance matrix $\hat{\mathbf{K}}_{\text {inst }}$ is computed with $J$ snapshots (centered on the lth snapshot):

$$
\hat{\mathbf{K}}_{\mathrm{inst}}=\sum_{j=l-J / 2}^{l+J / 2} \vec{x}_{j} \vec{x}_{j}^{H} .
$$


The $M$ principal eigenvectors ${ }^{16,17}$ of this instantaneous covariance matrix are then used to estimate $\Phi$, the instantaneous interference subspace: $\Phi_{\text {data }}=\left[\vec{u}_{1} \vec{u}_{2} \cdots \vec{u}_{M}\right]$, where $\left\{\vec{u}_{i}\right\}_{i=1}^{M}$ are the principal eigenvectors of $\hat{\mathbf{K}}_{\text {inst }}$. The success of the data-based method relies on the assumption that the interference and target subspaces are distinct in eigenvector space; specifically, it is assumed that that interferer comprises the only strong source in the data. Problems arise if the target is loud relative to the interferer (in which case the target will be part of the interference estimate and will itself be filtered). The data-based method also assumes that the interferer can be estimated well with $J$ snapshots; problems arise if the interferer still moves significantly during the computation of Eq. (22).

\section{Model-based interference filtering}

Model-based, time-varying interference filtering (MTIF) ${ }^{19-21}$ constructs its spatial filter assuming that some prior knowledge of the interferer's position is available. An example might be the tracking of a merchant ship by an airborne asset in the region. In order to estimate the interference subspace, a propagation model is used to determine the acoustic signature (replica vector) for an interferer at the given position. To protect against inaccuracies in the interferer position, the interference subspace is computed using $M$ replica vectors spanning a spatial region centered at the instantaneous position estimate of the interferer: $\Phi_{\text {model }}$ $=\left[\vec{v}\left(\Theta_{0}\right) \vec{v}\left(\Theta_{1}\right) \cdots \vec{v}\left(\Theta_{M-1}\right)\right]$, where $\Theta_{1}, \ldots, \Theta_{M-1}$ represent neighboring coordinates in range and depth (and possibly bearing) to the center coordinate $\Theta_{0}$. The amount of range and depth "padding" that is necessary is determined by the accuracy of the prior knowledge (the greater the uncertainty in the position of the interferer, the larger $M$ should be); any padding increases the size of the interference subspace estimate. Padding is also necessary when environmental information is uncertain, in which case more replica vectors are needed to describe a given interferer. Clearly, this method is dependent on accurate external information, but it does not make any assumptions about interferer versus target strength (in contrast to the data-based method).

\section{Hybrid interference filtering}

The hybrid method of interference filtering combines information from both the data-based and model-based approaches. To accomplish this, the model-based replica vectors $\left\{\vec{v}_{m}\right\}_{m=1}^{M}$ that span the presumed interferer position are projected onto the data-based interference subspace spanned by the principal eigenvectors of $\hat{\mathbf{K}}_{\text {inst }}$. The hybrid interference subspace is then formed by selecting only those replica vectors with significant projection onto the data: $\Phi_{\text {hybrid }}$ $=\left\{\vec{v}_{m}\right\}, \vec{v}_{m}^{H} \Phi_{\text {data }} \geqslant \gamma$, where $\gamma$ is an adjustable parameter between 0 and 1 representing the minimum acceptable projection. Higher values of $\gamma$ produce more conservative interference subspace estimates; in the examples below, $\gamma=0.6$, requiring fairly (but not extremely) high correlation between replicas in the model subspace and the data. In general, the greater one's confidence in the model (versus the data), the lower $\gamma$ should be. Note that the hybrid method essentially uses $\Phi_{\text {model }}$ except where the interference model is deemed inaccurate (as measured by correlation with $\Phi_{\text {data }}$ ).

\section{RESULTS}

In this section, the three AMFP motion mitigation techniques proposed earlier are applied to data from the Santa Barbara Channel Experiment (SBCX). Baseline AMFP performance is computed using the MVDR-DL weight vector in Eq. (3).

Results are presented in the form of MFP ambiguity surfaces (or derivatives), with the implicit assumption that detection is done in the spatial domain. Note that because of the redundancy in MFP steering vectors, it is inherently difficult to perform detection on MFP ambiguity surfaces. Performance is quantified by output SBNR (signal-tobackground-and-noise ratio), which for an ambiguity surface is calculated as the ratio (in $\mathrm{dB}$ ) of the output signal peak relative to the level of the background (consisting of output noise, interference, and possibly strong source sidelobes). The background level is defined as the 25 th percentile of the ordered output powers on the ambiguity surface. For strong discrete sources, the 25th percentile measure may reflect source sidelobes, which are not traditionally considered background. However, it should be pointed out that for detection in the spatial domain, discrete interferer sidelobes often comprise the "background," especially for data taken in heavy-shipping-density environments where interferers are constantly present. Thus, a processor that lowers sidelobes not only improves localization but also improves detection in the spatial domain, and this is reflected in both the 25 th percentile measure and in the resulting SBNR.

The SBCX experiment was conducted in April 1998 in the 200-m-deep littoral waters of the Santa Barbara Channel. ${ }^{22}$ One of the passive acoustic sensors deployed during the experiment was a 150-hydrophone volumetric array called the full-field processing (FFP) array. The FFP array consisted of five 30-phone VLAs arranged in a pentagonal configuration. The combination of vertical and horizontal aperture allowed full, three-dimensional localization in range, depth, and bearing.

One of the acoustic sources deployed during SBCX was a J15-3 transducer that was towed by a research vessel, the Acoustic Explorer (AX). The J15-3 was used to generate a comb sequence of 12 tones at approximately $159 \mathrm{~dB}$ re 1 $\mu \mathrm{Pa}$ source level. The AX contained an onboard GPS receiver for platform position information, and a nearby radar station produced track information for surface vessels in the SBCX area. $^{22}$ The SBCX site bathymetry is shown in Fig. 1. Replica vectors were computed by using the KRAKEN normal mode program $^{30}$ to generate mode functions and wavenumbers for water depths ranging from 50 to $260 \mathrm{~m}$. A twodimensional adiabatic approximation was then applied to derive range-dependent replica vectors (with the range dependence due to varying site bathymetry). The replicas were computed using a geoacoustic model that consisted of two sediment layers overlaying an acoustic half-space; the parameters used for the sediment were obtained from previous investigation in this area and are given in Table I. The 


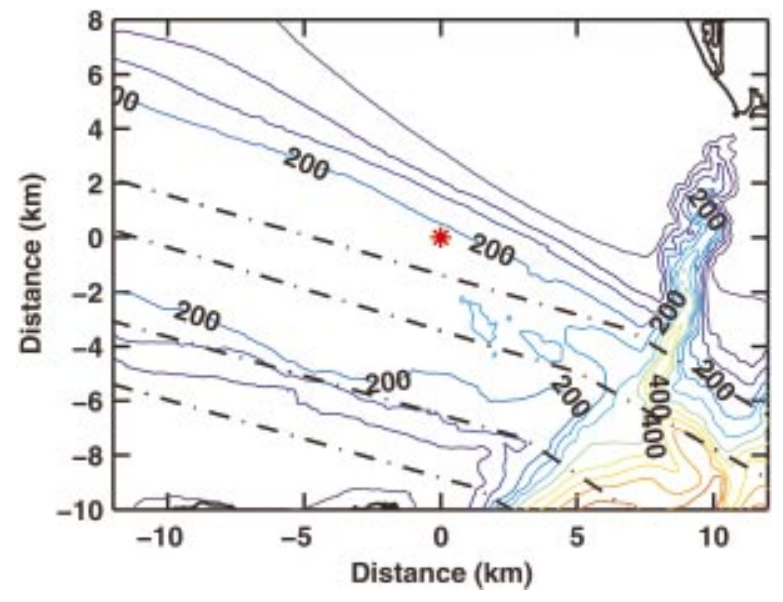

FIG. 1. SBCX site bathymetry. Dashed lines indicate shipping lanes. The map origin is the FFP array, indicated by the red asterisk. The California coastline is in the northeast corner of the map.

sound speed profile (SVP) for the water column was measured experimentally in several regions throughout the duration of the experiment; the replicas were computed using an average SVP shown in Fig. 2.

Figure 3 shows processing of data from a single VLA ( $N=30$ phones) for $300 \mathrm{~s}$ at $235 \mathrm{~Hz}$, one of the comb frequencies. Time-averaged covariance estimation was used with $\Delta t=1 \mathrm{~s}$, resulting in 300 snapshots over $300 \mathrm{~s}$. CMFP and AMFP output were computed using the CMFP weight vector in Eq. (2) and the AMFP weight vector in Eq. (3), respectively, and the results are shown in Figs. 3(a) and (b). Note that the $90.4 \mathrm{~dB}$ peak of the CMFP surface, according to the sonar equation, equals SL-TL-MM, with source level $\mathrm{SL}=159 \mathrm{~dB}$, transmission loss $\mathrm{TL} \sim 60 \mathrm{~dB}$ for a $2 \mathrm{~km}$ source, and mismatch $\mathrm{MM} \sim 8.4 \mathrm{~dB}$ due to both motion and steering vector mismatch. The AMFP peak of $87.4 \mathrm{~dB}$ is lower because of greater mismatch. The CMFP output in Fig. 3(a) displays the characteristically high sidelobes of nonadaptive MFP processing; this is manifested by the measured background level of $84 \mathrm{~dB}$, resulting in an output SBNR of $6.4 \mathrm{~dB}$. The AMFP output in Fig. 3(b) displays significantly lower sidelobes than CMFP; the background level is $76.3 \mathrm{~dB}$ and the SBNR is $11.1 \mathrm{~dB}$. However, the motion of the target over $300 \mathrm{~s}$ (about $780 \mathrm{~m}$ in range) has resulted in peak dispersion; the motion loss from Eq. (10) is $\sim 7.3 \mathrm{~dB}$ (vs $5.8 \mathrm{~dB}$ for CMFP). Motion loss is greater for AMFP than for CMFP because of the higher resolution of the adaptive weight vectors.

Figure 4 demonstrates motion mitigation via MBRR

TABLE I. Parameters for geoacoustic model used in SBCX data: $z=$ depth from surface; $\quad c_{c}=$ compressional sound speed; $\rho=$ density; $\alpha_{c}=$ compressional wave attenuation.

\begin{tabular}{cccc}
\hline \hline$z(\mathrm{~m})$ & $c_{c}(\mathrm{~m} / \mathrm{s})$ & $\rho\left(\mathrm{g} / \mathrm{cm}^{3}\right)$ & $\alpha_{c}(\mathrm{~dB} / \lambda)$ \\
\hline 209 & 1607 & 1.95 & 0.37 \\
309 & 1702 & 1.95 & 0.37 \\
309 & 1862 & 1.98 & 0.035 \\
609 & 2374 & 1.98 & 0.035 \\
609 & 2374 & 2.03 & 0.04 \\
\hline \hline
\end{tabular}

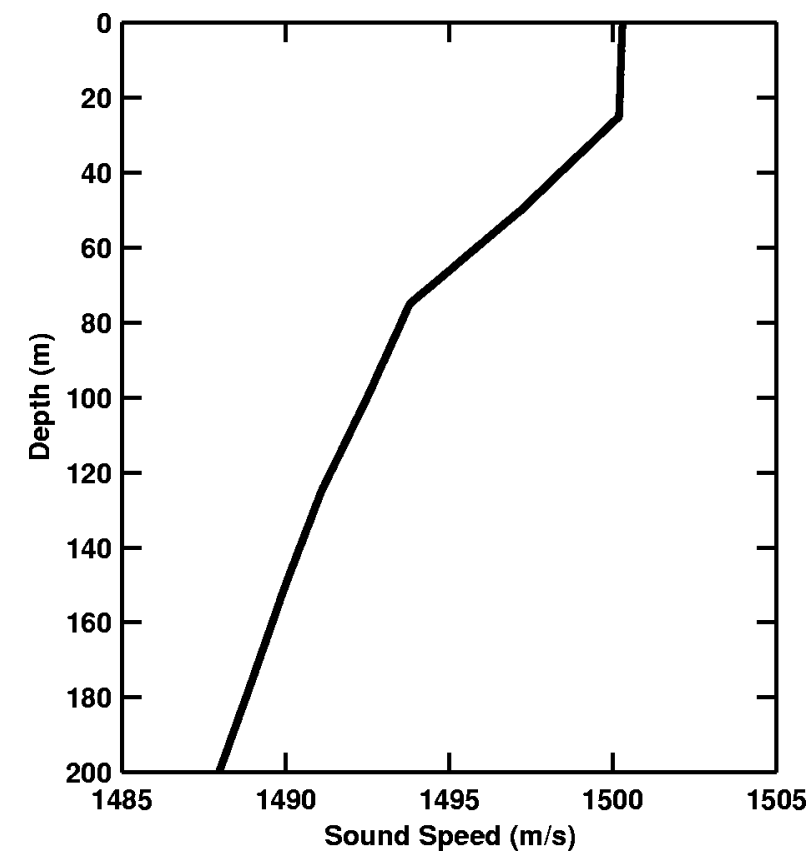

FIG. 2. Typical shallow-water, downward-refracting sound speed profile (SSP) used in all KRAKEN computations and obtained from averaging multiple measurements.

(mode-based rank reduction). First, Figs. 4(a) and (b) show eigenvector-based and mode-based AMFP rank reduction, respectively, for the full $300 \mathrm{~s}$ processed in Fig. 3. The eigenvector-based rank reduction in Fig. 4(a) is achieved using dominant mode rejection (DMR) with rank 10 (compared to the array size of 30). For the long observation time, snapshots are plentiful (300 snapshots for $\Delta t=1 \mathrm{~s}$ FFT window), so rank reduction is not necessary for computational purposes. Indeed, as has been observed previously by the authors, ${ }^{7,29}$ reduced-rank DMR displays poorer sidelobe rejection than the full-rank MVDR-DL. The result, in this case, is that the background level increases by $3.1 \mathrm{~dB}$ over the full-rank MVDR-DL to $79.4 \mathrm{~dB}$, and the output SBNR decreases by $3 \mathrm{~dB}$ to $8.1 \mathrm{~dB}$. For the long observation time, then, there is no reason to perform eigenvector-based rank reduction.

By contrast, MBRR is highly effective over the long observation time because of wider cell-widths in the reduced-rank mode space. Figure 4(b) shows MBRR achieved by retaining the ten lowest-order modes. The range resolution for the reduced-rank mode result-estimated from Eq. (6), but with $k_{M}$ replaced by $k_{P}$, the highest propagating mode in the reduced-rank subspace-is approximately $346 \mathrm{~m}$, compared to the $68 \mathrm{~m}$ resolution of the full-rank result. The result of this wider cell-width is a higher target peak (90.3 dB compared to $87.4 \mathrm{~dB}$ in the full-rank result) and a $4.4 \mathrm{~dB}$ increase in output SBNR (15.5 to $11.1 \mathrm{~dB}$ ), because the target has transited fewer MFP cells over the $300 \mathrm{~s}$ observation time and there is correspondingly less motion loss [5.2 dB compared to $7.3 \mathrm{~dB}$ for the full-rank case, using Eq. (10)]. Note that basic sonar equation computations (assuming that transmission loss follows a cylindrical spreading law) dictate that a $3 \mathrm{~dB}$ increase in output SBNR results in a doubling of detection range; thus, the $4.4 \mathrm{~dB}$ 

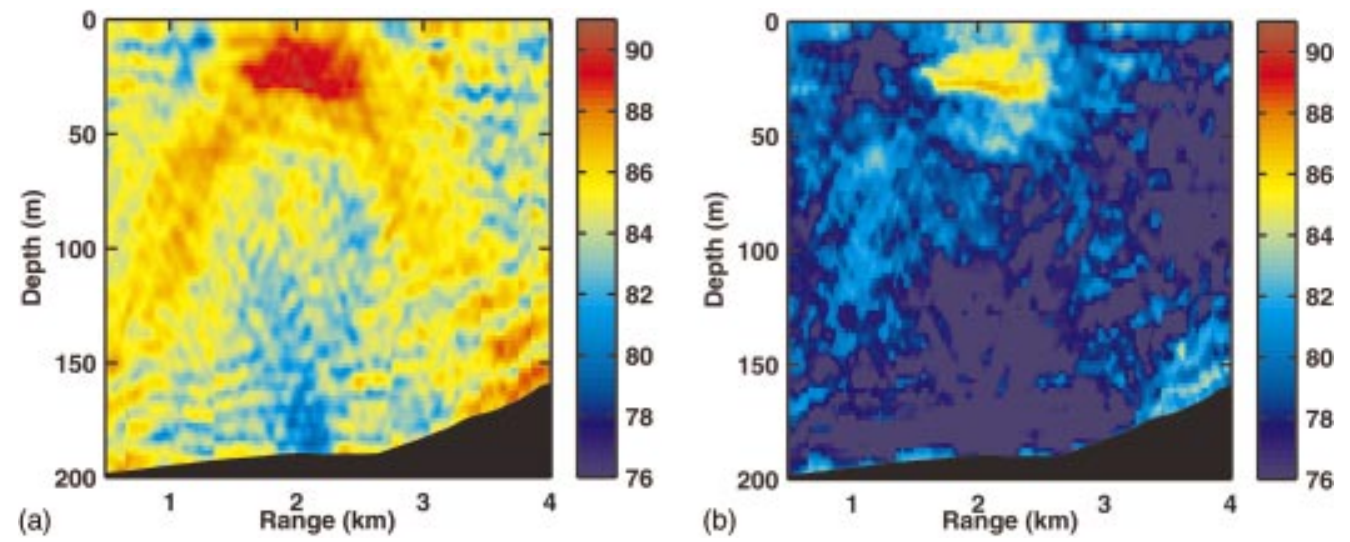

FIG. 3. Ambiguity surfaces from VLA data ( $N=30$ phones) for a $235 \mathrm{~Hz}$ tone processed with an observation time of $T=300 \mathrm{~s}$ and FFT window $\Delta t=1 \mathrm{~s}$. The range of the AX (according to GPS) was 1.67-2.45 km from the array. Nonadaptive CMFP output in (a) displays characteristically high sidelobes, with output SBNR $6.4 \mathrm{~dB}$. Adaptive MVDR-DL output in (b) shows lower sidelobes but a dispersed peak due to target motion; output SBNR is $\sim 11.1 \mathrm{~dB}$. Estimated target motion loss is $7.3 \mathrm{~dB}$ due to the $0.78 \mathrm{~km}$ motion of the target in range during the $300 \mathrm{~s}$ observation time. Colorbar units are $\mathrm{dB} r e 1 \mu \mathrm{Pa} / \mathrm{Hz}$.

increase in output SBNR is significant. Figure 4(b) vividly illustrates, then, the motion mitigation over long observation times provided by adaptive MBRR.

The more typical application of rank reduction however, involves very short time intervals over which source motion is less pronounced. Figures 4(c) and (d) show reduced-rank
AMFP output over $11 \mathrm{~s}$ (11 snapshots) of data within the original $300 \mathrm{~s}$ observation period; during these $11 \mathrm{~s}$, the target transited less than one full range cell-width (resulting in little motion loss). Eigenvector-based rank reduction using rank-10 DMR in Fig. 4(c) performs poorly because there are too few snapshots to effectively reject all sidelobes in eigen-
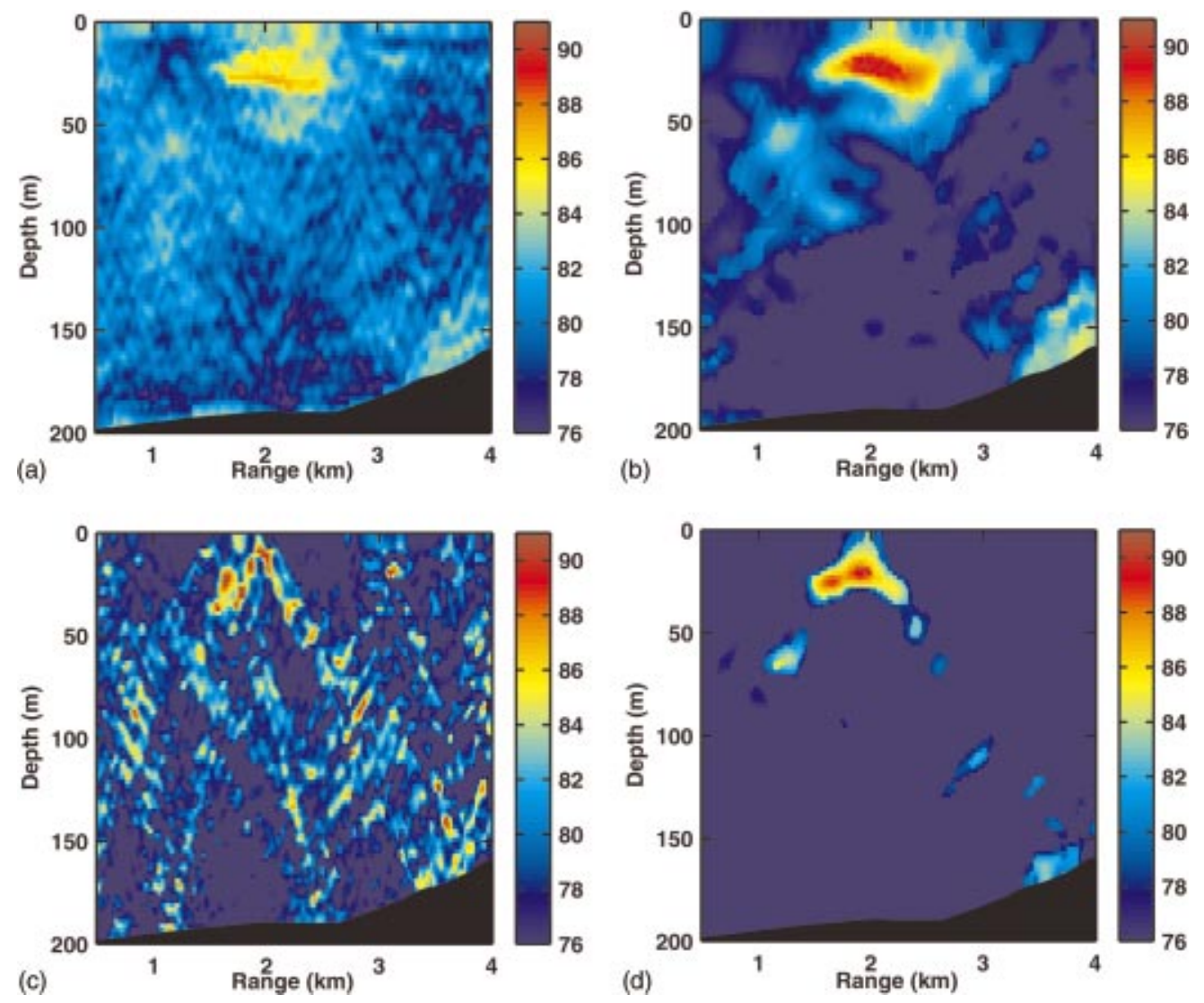

FIG. 4. Reduced-rank AMFP surfaces for the same data as in Fig. 3. (a) and (b) Rank-10 DMR and Rank-10 mode space AMFP for $T=300 \mathrm{~s}$ of data. Rank-10 DMR in (a), as expected, has higher sidelobes than full-rank MVDR-DL in Fig. 3(b), resulting in lower output SBNR of 8.1 dB. Rank-10 mode space in (b) is less sensitive to motion (because of wider MFP cells in reduced mode space), resulting in less motion loss (5.2 dB vs $7.3 \mathrm{~dB}$ for the full-rank case), a higher signal peak (almost $3 \mathrm{~dB}$ better than full-rank MVDR), and output SBNR of $15.5 \mathrm{~dB}$. (c) and (d) Rank-10 DMR and Rank-10 mode space AMFP for $T$ $=11 \mathrm{~s}$ (11 snapshots) of data. Rank-10 DMR in (c) is ineffective because the reduced-dimension eigenvalue spectrum no longer allows adequate sidelobe nulling; the output SBNR is $16.9 \mathrm{~dB}$, but there are multiple peaks within $3 \mathrm{~dB}$ of the target peak. Rank-10 mode space in (d) is highly effective because significant filtering occurs during modal rank reduction; the background level drops to $67 \mathrm{~dB}$, resulting in an output SBNR of $22.4 \mathrm{~dB}$. Colorbar units are $\mathrm{dB}$ re $1 \mu \mathrm{Pa} / \mathrm{Hz}$. 
vector space. Even though the measured output SBNR is high $(16.9 \mathrm{~dB})$, there are several "false" peaks of nearly the same power as the main target peak, so the target has not been unambiguously detected. By contrast, the rank-10 mode-space result (ten lowest-order modes retained) in Fig. 4(d) displays excellent sidelobe rejection and an unambiguous target peak (output SBNR $\sim 22.4 \mathrm{~dB}$ ). The reason for this is twofold: first, the ten lowest-order modes represent all the significant propagating modes at the $235 \mathrm{~Hz}$ frequency, so rank-10 AMFP output is still meaningful in mode space; second, the wider cell-widths in the reduced-rank mode space effectively "group" neighboring ambiguity peaks into larger peaks regions. Figure 4(d) illustrates that when computing reduced-rank AMFP output over very short observation intervals to mitigate motion, mode space provides a physically meaningful and intelligent way to perform the rank reduction.

Over both short and long observation times, the distinguishing feature for adaptive MBRR is its wider cell widths. This can be advantageous, since the coarser MFP cells reduce the number of "beams" that need to be formed to cover a given search region adequately. However, the coarser MFP cells also result in poorer localization, making it more likely for interferers to reside within one cell width of the target and thus appear in the same peak region as the target. The latter is not as great a concern as it may appear, given the potential with MFP for range, depth, and bearing discrimination.

It is important to note that the success of mode-based processing requires enough environmental information to compute the modal decomposition in Eq. (14) accurately. The SBCX environment was fairly well characterized, but not extraordinarily so, as the mode functions in the abovediscussed results were computed using historical geoacoustic parameters (Table I) and an average SVP (Fig. 2), neither of

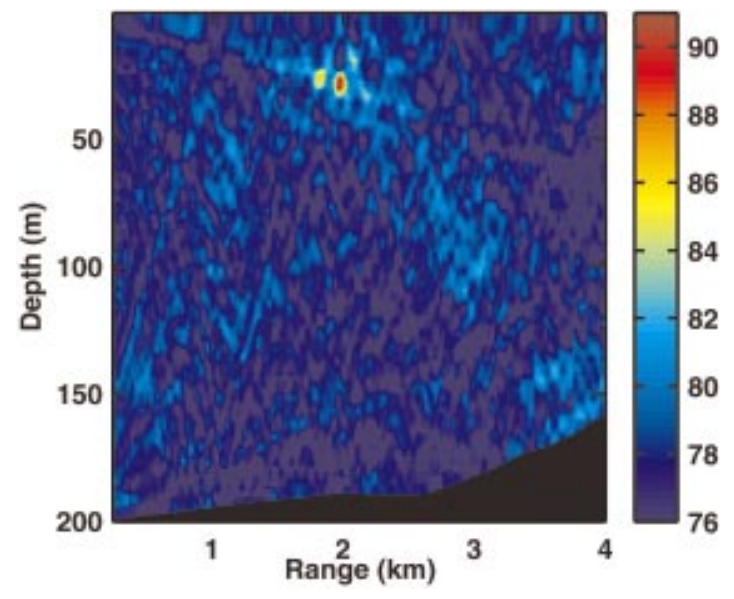

FIG. 5. Target-motion-compensated AMFP output for the same data as in Fig. 3, also processed for observation time $T=300 \mathrm{~s}$. The plot shows the signal focus that results when motion compensation is applied using the GPS track with a focus range of $2.0 \mathrm{~km}$ from the array. Target motion compensation adjusts the amplitude and phase of each snapshot according to a given velocity hypothesis. The signal peak is $91.1 \mathrm{~dB}$ [compared to 87.4 $\mathrm{dB}$ for uncompensated MVDR output in Fig. 3(b)], resulting in an output SBNR of $14.4 \mathrm{~dB}$. Colorbar units are $\mathrm{dB}$ re $1 \mu \mathrm{Pa} / \mathrm{Hz}$.

which is entirely accurate for the SBCX data. Thus, it can be reasonably stated that mode-space processing is only somewhat sensitive to environmental mismatch. Computing the modal decomposition accurately also requires an array capable of resolving the modes; the SBCX VLA, which is almost fully spanning, is an example of the latter.

In contrast to the motion mitigation provided by reduced-rank mode-based processing, ETMC (explicit target motion compensation) as detailed in Sec. III C uses target track hypotheses to correct for target motion explicitly. Figure 5 shows ETMC AMFP output over the $300 \mathrm{~s}$ observation interval processed in Fig. 3. The result is a focused target
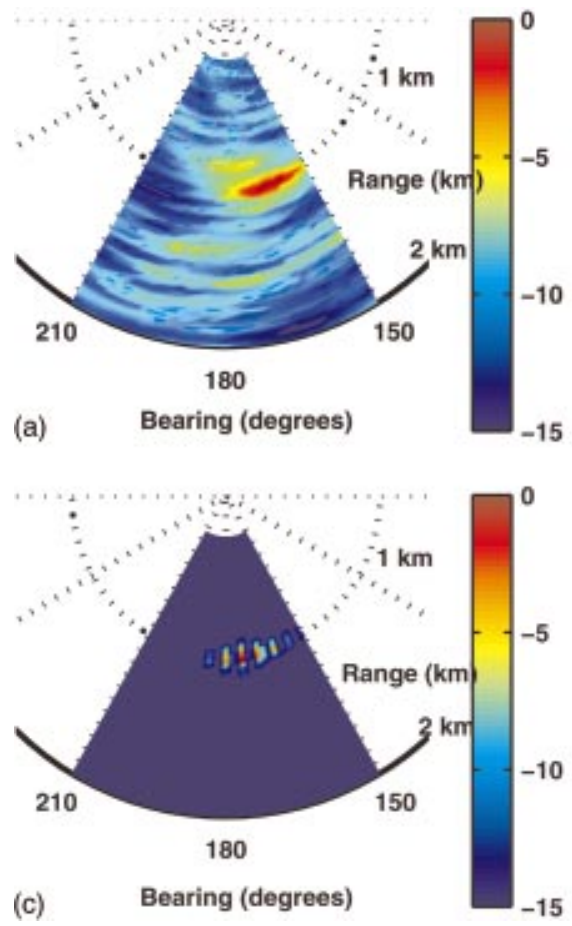
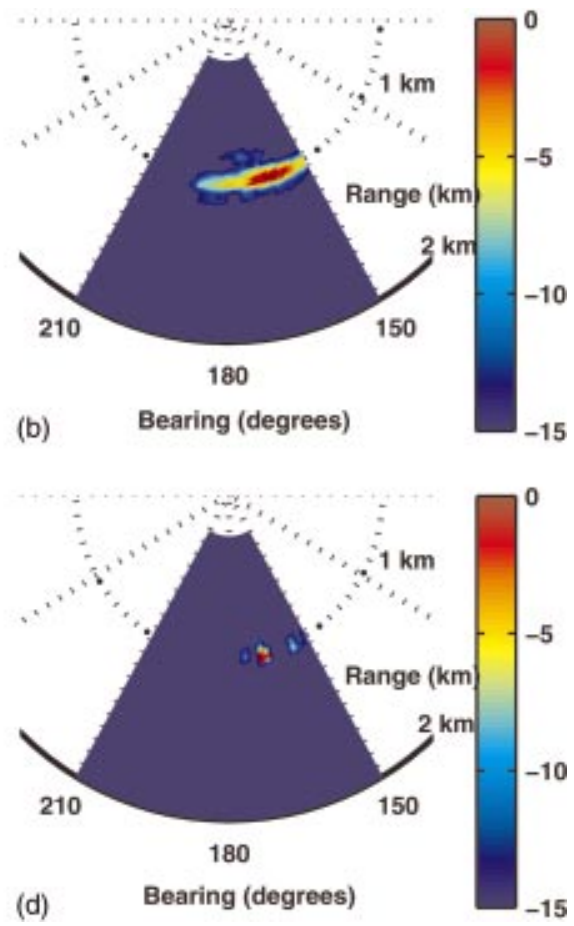

FIG. 6. Target motion compensation in bearing, applied to data from multiple VLAs. Results are range-bearing ambiguity surfaces for 1-3 VLAs processed coherently on a $235 \mathrm{~Hz}$ tone over observation time $T=120 \mathrm{~s}$, using FFT window $\Delta t=1 \mathrm{~s}$. The single VLA result in (a) shows coarse bearing localization due to the tilt of the VLA. Coherent processing of two VLAs should give additional azimuth resolution due to the $130 \mathrm{~m}$ horizontal baseline, but the uncompensated result in (b) is equivalent to incoherent array averaging because of motion decorrelation effects. Motion-compensated results for two VLAs (c) and three VLAs (d) give the expected azimuthal resolution and full, coherent array gain. 

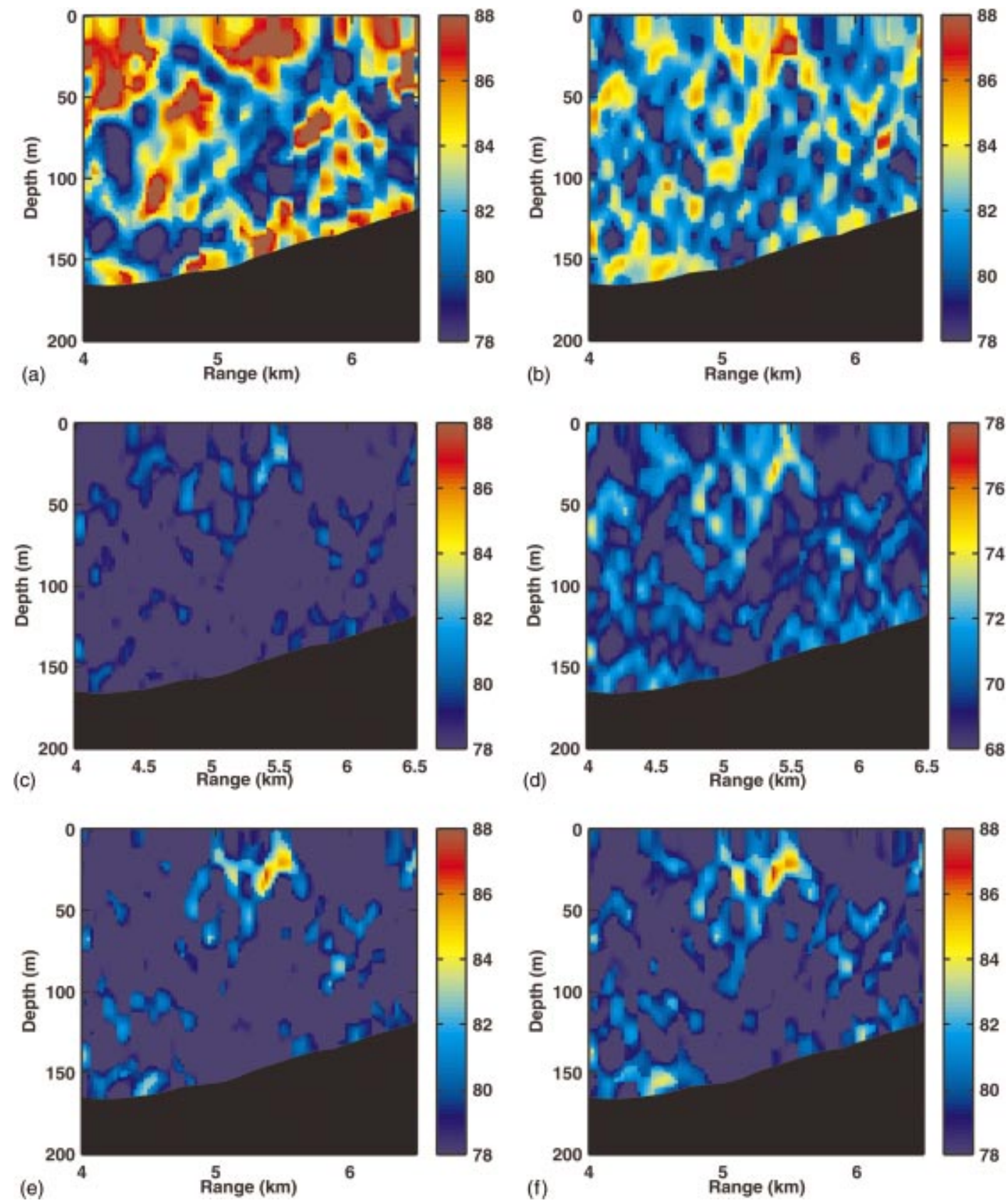

FIG. 7. Time-varying interference filtering of AMFP output at $94 \mathrm{~Hz}$ (time-averaged covariance matrix, $T=200 \mathrm{~s}, \Delta t=1 \mathrm{~s}$ ). The AX was $5.05-5.75 \mathrm{~km}$ from the array at the same time a surface ship was traveling in the eastbound shipping lane ( $\sim 6.7 \mathrm{~km}$ from the array), with estimated interference level $163 \mathrm{~dB}$. (a) The unfiltered result with output SBNR $7.8 \mathrm{~dB}$; however, the target is not unambiguously detected because of interferer sidelobes. (b) The result after motion compensation only (output SBNR remains $7.8 \mathrm{~dB}$ but the target is localized). (c) Data-based filtering followed by motion compensation, with the principal eigenvector used to estimate the instantaneous interference subspace; the resulting output SBNR is $6.8 \mathrm{~dB}$, a decrease from the unfiltered result due to undesired target filtering. (d) Data-based filtering with two principal eigenvectors; the resulting output SBNR is again $6.8 \mathrm{~dB}$, but altogether too much of the target has been filtered (target peak $12.2 \mathrm{~dB}$ below the unfiltered result-note the lower colorbar). (e) Model-based filtering followed by motion compensation, with $M=7$ replica vectors used to estimate the instantaneous interference subspace; the resulting output SBNR is $11.2 \mathrm{~dB}$. (f) Hybrid filtering, with $M=7$ and $\gamma=0.6$; the resulting output SBNR is $10.8 \mathrm{~dB}$. Both model-based and hybrid filters produce good results for this scenario because the interferer position was approximately known (from a radar track) and the acoustic propagation model was accurate. Colorbar units are $\mathrm{dB}$ re $1 \mu \mathrm{Pa} / \mathrm{Hz}$.

peak (91.1 dB, compared to $87.4 \mathrm{~dB}$ for the uncompensated AMFP result) and output SBNR improvement of $3.3 \mathrm{~dB}$ $(11.1$ to $14.4 \mathrm{~dB})$. Note that in this particular example, the true target track was approximately known, so the output is close to a best-case result. Further research is needed to determine the sensitivity of the motion compensation algorithm to target track accuracy.

A second example of ETMC, this time in the bearing dimension, is illustrated in Fig. 6. In this example, range- bearing AMFP output is shown for data from multiple VLAs (recall that the FFP array in SBCX contained five VLAs), processed at $235 \mathrm{~Hz}$ over $T=120 \mathrm{~s}$, using a $\Delta t=1 \mathrm{~s}$ FFT window. For a single VLA [Fig. 6(a)], the array tilt of the VLA (approximately $15^{\circ}$ ) allows coarse azimuthal localization. The addition of a second VLA [Fig. 6(b)] should provide finer resolution and coherent array gain, but target motion-the differential Doppler across the two VLAsintroduces a time-varying phase that prevents any coherent 
processing of the two VLAs; the result is then an incoherent sum of single-VLA outputs (with the same target peak region). Note that the motion compensation expression in Eq. (21) contains a phase correction that accounts for the modally dependent Doppler signatures. After correcting for this phase, the compensated surface for two VLAs [Fig. 6(c)] shows the grating pattern one would expect from a sparse horizontal aperture. Processing of three VLAs with motion compensation [Fig. 6(d)] results in a single strong peak with array gain $10 \log _{10} 3 \approx 5 \mathrm{~dB}$ higher than the single-VLA result. It is important to note once again that ETMC is designed for a single source and that the SBNR improvement seen in the above two examples is not guaranteed for multiple sources.

All of the results to this point have demonstrated improved AMFP output on a single, moving target. For data that also include an interferer, the third motion mitigation technique is necessary, that of MTIF (model-based, timevarying interference filtering). Figure 7 shows AMFP output for data containing a loud, moving interferer located $\sim 6.7$ $\mathrm{km}$ from the FFP array (at another bearing) in addition to the AX towed source located $\sim 5.5 \mathrm{~km}$ from the array. The processing was done at frequency $94 \mathrm{~Hz}$ (another of the comb frequencies), with observation time $T=200 \mathrm{~s}$ and FFT window $\Delta t=1 \mathrm{~s}$. The basic AMFP result in Fig. 7(a) is contaminated by sidelobes from the loud interferer, to the point that the target is not detectable. The measured output SBNR is $7.8 \mathrm{~dB}$, but there are several peaks higher than the target peak (at $5.5 \mathrm{~km}$ range and $15 \mathrm{~m}$ depth). Target motion compensation [Fig. 7(b)] focuses target energy to the correct location, but sidelobes from the interferer remain; the output SBNR remains $7.8 \mathrm{~dB}$, but the only peak of the ambiguity surface is the target. In Figs. 7(c) and (d), data-based interference filtering using one and two principal eigenvectors, respectively, is used to remove the interferer sidelobes. Because the target and the interferer have nearly the same phone-level (source level minus transmission loss) power in this example, the target has been incorporated into the eigenvector estimate of the interferer subspace, resulting in undesired filtering of the target; the resulting output SBNR of 6.8 $\mathrm{dB}$ for both Figs. 7(c) and (d) is actually lower than the unfiltered result. By contrast, Fig. 7(e) demonstrates the use of MTIF to remove the interferer sidelobes. The MTIF (model-based) result uses a rough estimate of the interferer position (derived here from a radar track) to generate a timevarying, location-based null on the interferer; the interferer space has dimension $M=7$, to account for potential inaccuracies in the interferer track information. Application of MTIF increases output SBNR to $11.2 \mathrm{~dB}$, an increase of 3.4 dB over the unfiltered result. In Fig. 7(f), hybrid interference filtering is used to remove the interferer sidelobes. The hybrid method uses the model-based interference subspace estimate (again with dimension $M=7$ ) but eliminates any replicas from the interferer space estimate that do not have high correlation with the principal eigenvectors of the data (as measured by the correlation parameter $\gamma=0.6$ ). The effect of this is greater protection of the target peak ( $87.6 \mathrm{vs} 86.6 \mathrm{~dB}$ for the model-based output) at the expense of less sidelobe rejection (background level approximately $1.5 \mathrm{~dB}$ higher than the model-based output). The output SBNR of $10.8 \mathrm{~dB}$ for the hybrid output is still $3 \mathrm{~dB}$ higher than that for the unfiltered output in Fig. 7(a). This example is a case where the model-based (and hybrid) interference filtering techniques perform well (because of accurate interferer track information) but the data-based filtering technique performs poorly (because the target is loud relative to the interferer).

There are cases, however, when the interferer track is unknown, so data-based interference filtering is the only option; the following example is one such case. Figure 8 shows range-time AMFP output derived from range-depth ambiguity surfaces by taking the maximum over a set of depths. Output was generated using FFT window $\Delta t=16 \mathrm{~s}$; the data contain both a loud, moving surface interferer and a weaker acoustic source at depth. Figure 8(a) is the basic "surface" AMFP result, generated from surface depths $0-20 \mathrm{~m}$. The track of the interferer is clearly visible, with $\sim 15 \mathrm{~dB}$ interferer-to-noise ratio at its closest point of approach, at 38 min. Figure 8(b) applies data-based interference filtering in which $M=2$ principal eigenvectors (computed using $J=7$ snapshots at each time instant) are used to generate the interferer null; the result is effective removal of nearly all interferer energy. Figure 8(c) is the basic "submerged" AMFP result, generated from depths 50 to $55 \mathrm{~m}$ that include the target depth. The target track is now visible, but it is still obscured by sidelobes from the surface interferer. Applying data-based interference filtering as above, in Fig. 8(d), results in effective removal of the interferer sidelobes and retention of the now-distinct target track. The SBNR improvement from applying data-based interference filtering at depth is up to $10 \mathrm{~dB}$ where the target and interferer tracks cross in range.

\section{CONCLUSIONS}

This paper proposed three techniques to improve AMFP performance for detecting quiet, moving targets. Each of the techniques was demonstrated on data examples to provide 3 $\mathrm{dB}$ or more improvement in output SBNR over basic AMFP output. Basic AMFP suffers both from target motion, in which the target moves across several resolution cells during the observation time and the target peak is reduced, and from interferer motion, in which the moving interferer occupies several adaptive degrees of freedom and reduces adaptive nulling capability.

The first motion mitigation technique, mode-based rank reduction, reduces the effects of both target and interferer motion over long observation times by increasing the effective size of the MFP resolution cells. Both target and interferers move across fewer resolution cells for a given observation time, and motion loss is correspondingly reduced. The technique was demonstrated on a data example in which target motion loss was reduced and output SBNR was increased by $4.4 \mathrm{~dB}$ compared to the basic, full-rank AMFP output. The technique is only somewhat sensitive to environmental mismatch, but it does require an array capable of resolving the acoustic modes (such as the SBCX VLA used in the data examples of this paper). Future work should examine the effectiveness of mode-based rank reduction for tactical horizontal line arrays. 

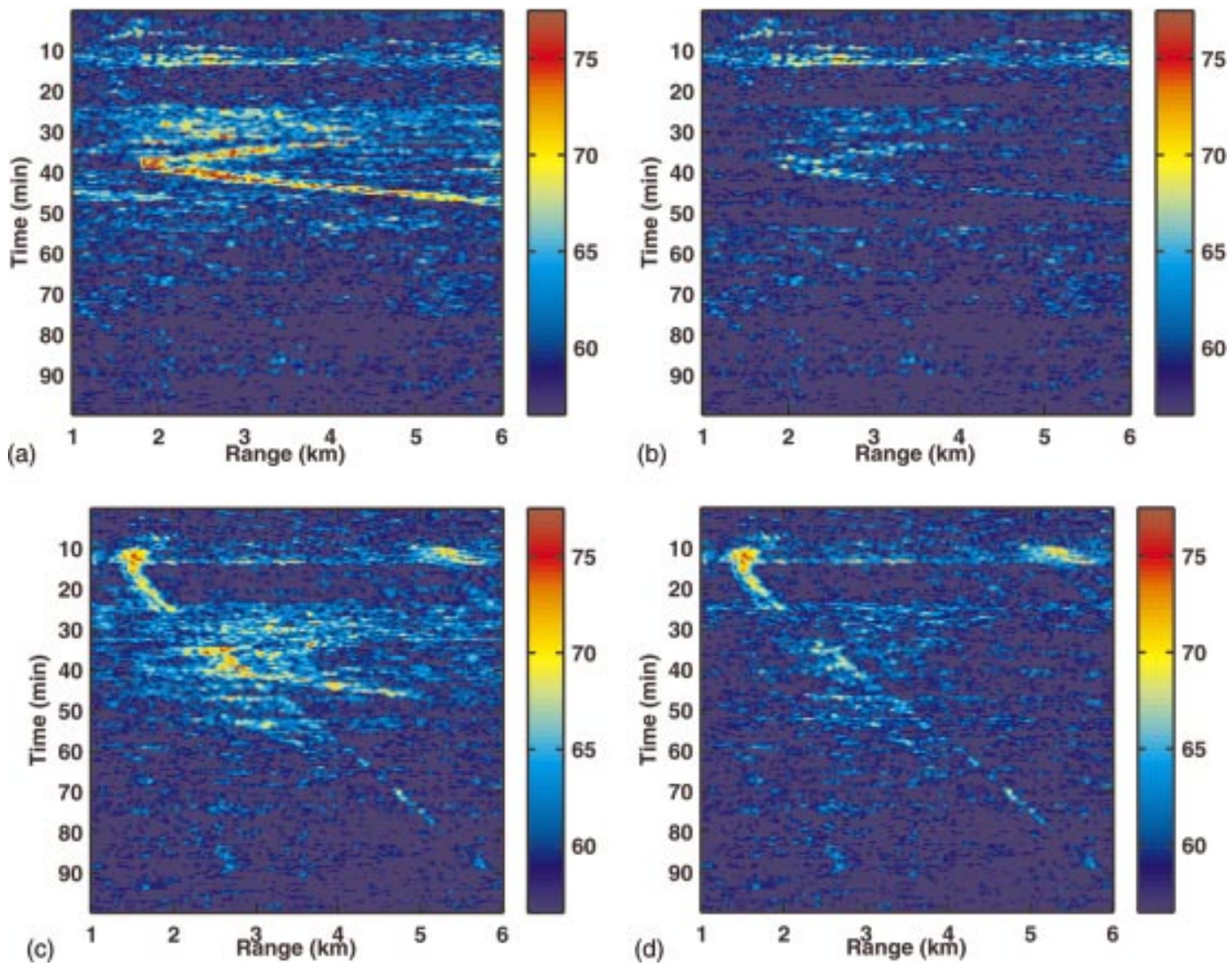

FIG. 8. AMFP range-time tracks, processed with FFT window $\Delta t=16 \mathrm{~s}$. Data include both a loud surface interferer and a weak acoustic source at depth, with the interferer approximately $30 \mathrm{~dB}$ louder. Plots (a) and (b) are derived from range-depth ambiguity surfaces focused at depths near the surface (less than 20 $\mathrm{m}$ depth). The data in (a) have not been filtered, and the ship track is clearly seen with INR at CPA of $15 \mathrm{~dB}$ (CPA is at $t=38$ min). Data-based filtering with $M=2$ eigenvalues and $J=7$ snapshots has been applied in (b), and the ship energy has been effectively removed. Plots (c) and (d) are derived from range-depth ambiguity surfaces focused at depths near the target depth of $50 \mathrm{~m}$. The data in (c) have not been filtered, and sidelobes from the surface interferer are still visible. After applying data-based filtering as in (b), the result in (d) removes the interferer sidelobes and retains the target track. SBNR improvement from data-based interference filtering is up to $10 \mathrm{~dB}$ where the tracks cross in range. Colorbar units are $\mathrm{dB} r e 1 \mu \mathrm{Pa} / \mathrm{Hz}$.

The second motion mitigation technique, explicit target motion compensation, reduces the effect of target motion over long observation times by using a hypothesized target track to focus data snapshots; done accurately, target motion compensation eliminates target motion loss (for a single target). This technique was demonstrated on the same data example to provide both improved output SBNR (3.3 dB better) and improved source localization over the basic, uncompensated AMFP result. Even greater SBNR gains are possible with more accurate target tracks. However, one of the open questions with this technique (and a focus for future work) is how sensitive it is to inaccuracies in the assumed target track and to environmental mismatch, both of which would reduce the effectiveness of the focusing operation. Also, it should be noted again that the technique is designed for a single source; another interesting question is how motion compensation might be combined with interference filtering to handle data with multiple sources.

The third motion mitigation technique, model-based time-varying interference filtering, reduces the effect of interference motion by placing a time-varying null on the interferer; done accurately, interference filtering removes interferer sidelobes entirely and increases output SBNR (potentially by a very large amount if the interferer is loud relative to the target). The model-based filtering technique was demonstrated on a data example in which a radar track was used to form an interference null based approximately on the location of the interferer, resulting in unambiguous detection of the target (not possible in the basic AMFP result) and a $3.4 \mathrm{~dB}$ increase in output SBNR over the basic, unfiltered AMFP output. Again, even greater SBNR gains are possible with more accurate interferer tracks. Greater protection of the target peak is achievable with the hybrid interference-filtering technique (combining elements of model-based and data-based filtering), at the expense of less interference nulling; hybrid filtering produced a $3 \mathrm{~dB}$ output SBNR increase in the same example. It is important to note that the use of external information for the model-based filtering technique removes the need to associate interferers with principal eigenvectors, which is a potential weakness of the data-based filtering method (when the target is loud relative to the interferer). However, accurate track and environmental information is needed for model-based filtering to succeed, and future work should investigate how sensitive the technique is to mismatch.

All three of the techniques presented here require intelligent application of external information. Mode-based rank reduction (the least dependent of the three techniques on external information) requires some knowledge of the number of significant propagating modes in the data. Target motion 
compensation requires an accurate target track hypothesis. And model-based interference filtering requires an accurate interferer track. To varying degrees, as mentioned earlier, all three techniques require accurate environmental information. Future work should examine the robustness of these techniques to inaccuracies in the assumed external information. ${ }^{31}$

\section{ACKNOWLEDGMENTS}

This work was sponsored by DARPA-ATO under Air Force Contract No. F19628-00-C-0002. Opinions, interpretations, conclusions, and recommendations are those of the authors and are not necessarily endorsed by the Department of Defense. The authors wish to thank Andrew Gronosky for help in formatting many of the figures in the paper and Brian Tracey for various technical consultations during the writing of the paper. The authors are also grateful for the efforts involved in the SBCX data collection under Newell Booth of SPAWAR, and for the efforts of Peter Daly of MIT and Robert Greene of SAIC in computing SBCX array shapes.

${ }^{1}$ A. Baggeroer, W. Kuperman, and P. Mikhalevsky, "An overview of matched field methods of ocean acoustics," IEEE J. Ocean. Eng. 18, 401424 (1993).

${ }^{2}$ J. Capon and N. Goodman, "Probability distributions for estimators of frequency wavenumber spectra," Proc. IEEE 58, 1785-1786 (1970).

${ }^{3} \mathrm{H}$. Cox, "Resolving power and sensitivity to mismatch of optimum processors," J. Acoust. Soc. Am. 54, 771-785 (1973).

${ }^{4}$ H. Cox, R. Zeskind, and M. Owen, "Robust adaptive beamforming," IEEE Trans. Acoust., Speech, Signal Process. 35, 1365-1376 (1987).

${ }^{5}$ A. Baggeroer and H. Cox, "Passive sonar limits upon nulling multiple moving ships with large aperture arrays," in Proceedings of the 33rd Asilomar Conference, 1999, pp. 103-108.

${ }^{6} \mathrm{H}$. Cox and R. Pitre, "Robust DMR and multi-rate adaptive beamforming," in Proceedings of the 31st Asilomar Conference, 1997, pp. 920-924.

${ }^{7}$ N. Lee, L. Zurk, and J. Ward, "Evaluation of reduced-rank, adaptive matched field processing algorithms for passive sonar detection in a shallow-water environment," in Proceedings of the 33rd Asilomar Conference, 1999, pp. 876-880.

${ }^{8}$ Y. Lee, P. Mikhalevsky, H. Freese, and J. Hanna, "Robust adaptive matched-field-processing," in Proceedings of the IEEE Oceans '93 Conference, 1993, pp. 387-392.

${ }^{9}$ E. Shang, C. Clay, and Y. Wang, "Source depth estimation in waveguides," J. Acoust. Soc. Am. 77, 1413-1418 (1985).

${ }^{10} \mathrm{~T}$. Yang, "A method of range and depth estimation by modal decomposition," J. Acoust. Soc. Am. 82, 1736-1745 (1987).

${ }^{11}$ T. Yang, "Effectiveness of mode filtering: A comparison of matched-field and matched-mode processing," J. Acoust. Soc. Am. 87, 2072-2084 (1990).

${ }^{12}$ H. Bucker, "Matched-field tracking in shallow water," J. Acoust. Soc. Am. 96, 3809-3811 (1994).

${ }^{13}$ L. Fialkowski et al., "Matched-field source tracking by ambiguity surface averaging," J. Acoust. Soc. Am. 110, 739-746 (2001).
${ }^{14}$ H. Hung and M. Kaveh, "Focussing matrices for coherent signal-subspace processing," IEEE Trans. Signal Process. 36, 1272-1281 (1988).

${ }^{15} \mathrm{~J}$. Krolik and D. Swingler, "Multiple broad-band source location using steered covariance matrices," IEEE Trans. Signal Process. 37, 1481 (1989).

${ }^{16}$ B. Freburger and D. Tufts, "Rapidly adaptive signal detection using the principal components inverse (PCI) method," in Proceedings of the 31st Asilomar Conference, 1997, pp. 765-769. The philosophy behind timevarying eigenvector filtering is essentially that of the so-called "principal component inverse" method, though the specific application of the filtering presented here, within the context of MVDR weight computation, is different.

${ }^{17}$ D. Tufts, R. Kumaresan, and I. Kirsteins, "Data adaptive signal estimation by singular value decomposition of a data matrix," Proc. IEEE 70, 684685 (1982).

${ }^{18}$ H. Cox, "Multi-rate adaptive beamforming (MRABF)," in Proceedings of the 2000 Sensor Array and Multichannel Signal Processing Workshop, Boston, MA, pp. 306-309.

${ }^{19}$ L. Zurk, N. Lee, and J. Ward, "Adaptive matched field processing for a moving target in a noisy shallow-water channel," in Proceedings of the 2000 Adaptive Sensor Array Workshop, Lexington, MA, pp. 103-107.

${ }^{20} \mathrm{~L}$. Zurk and J. Ward, "Model-based and data-based matched field processing of moving sources in a shallow water channel," J. Acoust. Soc. Am. 107, 2889 (2000).

${ }^{21}$ L. Zurk, N. Lee, and J. Ward, "Interference rejection for passive sonar using prior information with adaptive matched field processing," in Proceedings of the Fifth European Conference on Underwater Acoustics, Lyon, France, 2000.

${ }^{22}$ N. Booth, "Santa Barbara Channel Experiment (SBCX) Data Report," Space and Naval Warfare Systems Center, 1998.

${ }^{23} \mathrm{H}$. Bucker, "Use of calculated sound fields and matched field detection to locate sound sources in shallow water," J. Acoust. Soc. Am. 59, 368-373 (1976).

${ }^{24}$ B. Tracey, N. Lee, L. Zurk, and J. Ward, "Array design and motion effects for matched field processing," J. Acoust. Soc. Am. 108, 2645 (2000).

${ }^{25}$ L. Zurk, B. Tracey, and J. Munro, "Signal degradation mechanisms for passive localization of acoustic sources in shallow water," in Progress in Electromagnetic Research Symposium 2000, Cambridge, MA.

${ }^{26}$ I. Reed, J. Mallett, and L. Brennan, "Rapid convergence rate of adaptive arrays," IEEE Trans. Aerosp. Electron. Syst. 10, 853-863 (1974).

${ }^{27}$ N. Lee and N. Pulsone, "Performance of sample-covariance-based adaptive sonar detectors," in Proceedings of the 34rd Asilomar Conference, 2000, pp. 668-672.

${ }^{28}$ J. Ward, A. Baggeroer, and L. Zurk, "Rapidly adaptive matched field processing for nonstationary environments," in Proceedings of the 1998 Adaptive Sensor Array Workshop, Lexington, MA.

${ }^{29} \mathrm{~N}$. Lee, L. Zurk, and J. Ward, "Evaluation of reduced-rank, adaptive matched field processing for shallow-water target detection," in Proceedings of the 2000 Adaptive Sensor Array Workshop, Lexington, MA, pp. 25-30.

${ }^{30}$ M. Porter, "The KRAKEN normal mode program," Technical Report No. SM-245, SACLANT Undersea Research Centre, 1991.

${ }^{31}$ L. Zurk, N. Lee, and J. Ward, "3D adaptive matched field processing for a moving source in a shallow water channel," in Proceedings of the IEEE Oceans '99 Conference, Vol. 2, pp. 728-731. 